\title{
The molecular basis for sarcomere organization in vertebrate skeletal muscle
}

Zhexin Wang ${ }^{1}$, Michael Grange ${ }^{1}$, Thorsten Wagner ${ }^{2}$, Ay Lin Kho ${ }^{3}$, Mathias Gautel ${ }^{3}$ and Stefan Raunser ${ }^{2}$

${ }^{1}$ Max Planck Institute of Molecular Physiology, Dortmund, Germany, ${ }^{2}$ Max Planck Institute of Molecular Physiology, United States, ${ }^{3}$ King's College London, United States

Skeletal muscle is an essential tissue required for efficient movement in vertebrates. Individual muscle fibres can be very large syncytia comprised of the force-generating and load-bearing devices of muscles called sarcomeres. A precise molecular understanding of how the entire sarcomere is built is required to understand its role in health, disease and ageing. The current understanding of actin-myosin interactions and the function and mechanism of other essential sarcomeric proteins is derived from atomic structures of in vitro reconstituted components ${ }^{1-3}$, necessarily lacking important information in their biological context, or ensemble information derived from X-ray diffraction of muscle fibres ${ }^{4-6}$. Reconstructions of insect sarcomeres using conventional electron tomography ${ }^{7-9}$ and freeze-substituted samples ${ }^{10}$ gave insights into the three-dimensional structure of sarcomeres but were limited to low resolutions.

Here, we determine the in situ molecular architecture of vertebrate skeletal sarcomeres in the rigor state through electron cryo-tomography of cryo-focused ion beam-milled native myofibrils. The reconstructions reveal the three-dimensional organisation and interaction of actin and myosin filaments in the A-band, I-band and Z-disc at an unprecedented level of molecular detail. 3-dimensional annotation of the A-band and sub-volume averaging shows the interaction between myosin, tropomyosin and actin in molecular detail at $\sim 10 \AA$ resolution and reveals two coexisting conformations of actin-bound heads. The flexible orientation of the lever arm and the essential and regulatory light chains allows the two heads of the "double-headed" myosin not only to interact with the same actin filament but also to split between two actin filaments. Myosin heads bind to thin filaments in a pseudo-regular pattern: binding occurs roughly $37 \mathrm{~nm}$ apart due the orientation of actin subunits but the exact binding site on a thin filament is not strictly ordered. In the I-band, thin filaments mediate the transition from a hexagonal arrangement in A-band to a rhomboid pattern in Z-disc through irregular displacements. A shifts of tropomyosin position from the Mstate to the $\mathrm{C}$-state occurs at the $\mathrm{A} / \mathrm{I}$ junction.

In addition, different forms of Z-discs are observed with varying Z-disc thickness and angles between thin filaments and $\alpha$-actinin, behaving like a parallel hinge. 3D annotation of the Z-disc demonstrates how $\alpha$ actinin cross-links antiparallel actin filaments to form a mesh-like structure in the Z-disc. A prominent feature is a so-far undescribed doublet of $\alpha$-actinin cross-links with $\sim 6 \mathrm{~nm}$ spacing. The overall organisation of the Z-disc exhibits a heterogenous cross-link network, instead of a perfectly ordered lattice as previously conceived.

Collectively, our structure of the sarcomere, while not yet a complete atomic model of the entire structure, identifies previously unresolved details of the molecular architecture of the A-bands, I-bands and Z-discs and highlights the molecular plasticity of its components. It allows improved dynamic modelling of muscle contraction and will serve to test new concepts on the molecular,

cellular and physiological level, not least for the development of new approaches for muscle diseases.

\section{Acknowledgements}

We thank M. Saur for preliminary work and J. Mahamid and J. Plitzko for advice on cryo-FIB milling. We are thankful to S. Tacke for hardware optimization for cryo-FIB and R.S. Goody for critical reading 
of the written text. We are grateful to O. Hofnagel and D. Prumbaum for EM support and B. Brandmeier for technical assistance.
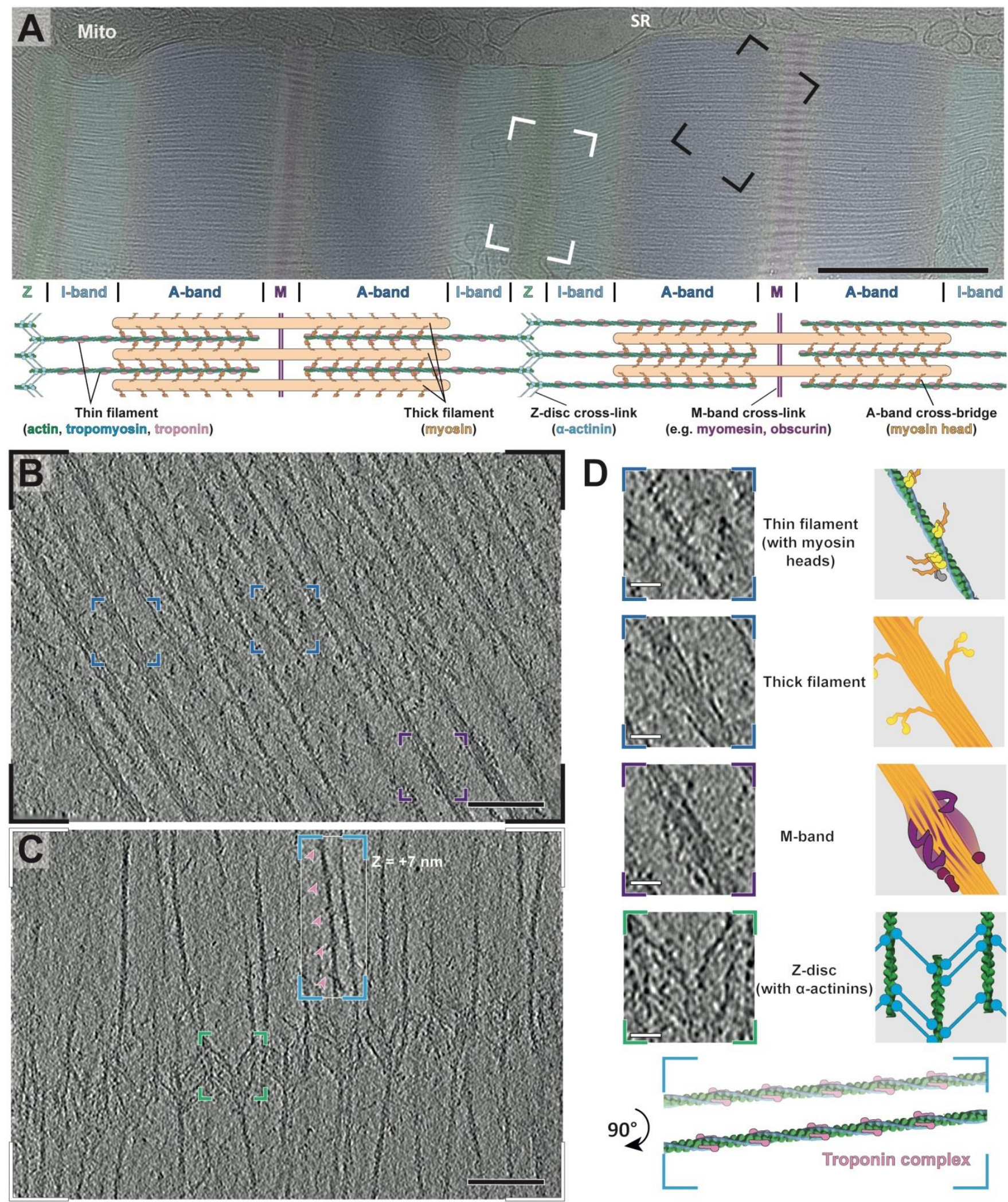
Figure 1. Figure 1. Isolated mouse skeletal myofibrils imaged using electron cryo-tomography.(A) Projection image of mouse skeletal muscle, with Z-disc, I-, M-, and A-bands visible (green, light blue, dark blue and purple, respectively). A schematic diagram is shown below, highlighting the lateral organisation and cross-links in different zones. Mitochondria (Mito) and sarcoplasmic reticulum (SR) can be identified between sarcomeres. Scale bar, $1 \mu \mathrm{m}$.(B) Slice through an electron cryo-tomogram spanning A- to M-band. Its representative position on a sarcomere is marked as a black box in (A) (not same sarcomere). In this region, myosin heads bound to the thin filament, myosin tails emanating from the thick filament (dark blue insets) and obscure protein densities at the M-band (purple inset) can be discerned. Scale bar, $100 \mathrm{~nm} .(\mathrm{C})$ Slice through an electron cryo-tomogram spanning I-band and Z-disc. Its representative position on a sarcomere is marked as white box in (A) (not same sarcomere). The tailfeather-like arrangement of a-actinin molecules cross-bridging thin filaments in a zig-zag manner is visible (green inset). Thin filaments in the I-band have regularly-spaced nodes corresponding to troponin complexes (pink arrow heads, blue inset). A slice of the same location but $7 \mathrm{~nm}$ above from the rest of the slice is shown inset. Scale bar, $100 \mathrm{~nm}$.(D) Larger view of insets described in (A-C), with cartoon depictions of densities. Scale bar, $20 \mathrm{~nm}$.
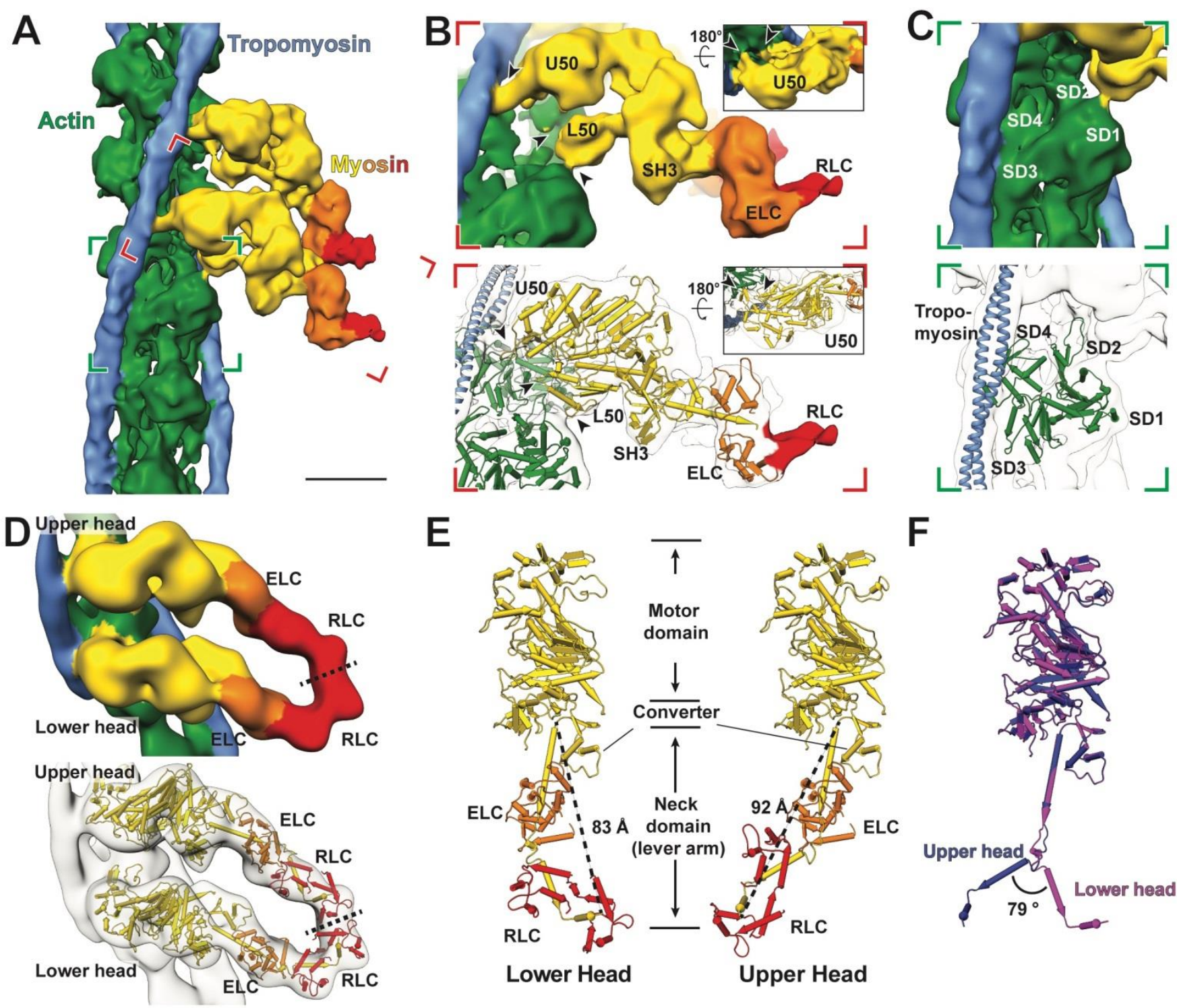

E
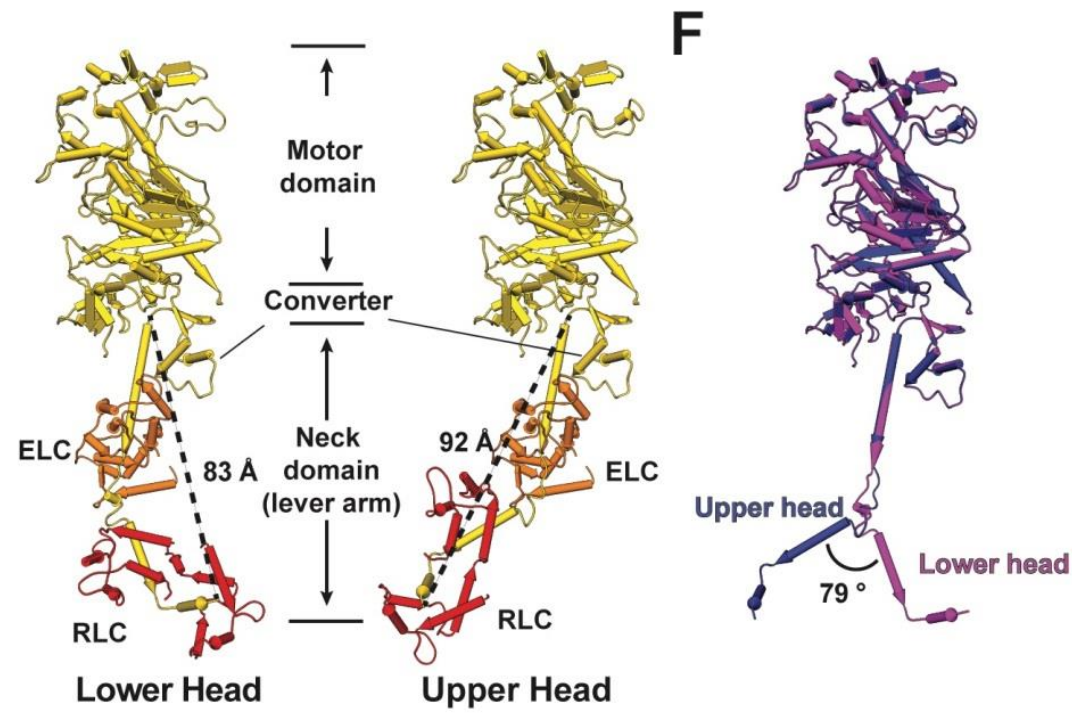
Figure 2. Figure 2. Sub-tomogram averaging of thin filaments reveals the interaction of a double-head myosin with the thin filament and two conformations of light chain domains within a double-head. (A) Surface view of the in situ actomyosin structure, showing actin filament (green), tropomyosin (blue) and myosin double heads, with motor domains (yellow), essential light chains (orange) and regulatory light chains (red). Scale bar, $5 \mathrm{~nm}$. (B) Close-up view of the lower myosin head and homology models based on PDBs 3I5G, 5JLH and 6KN8. The upper $50 \mathrm{kDa}$ (U50), lower $50 \mathrm{kDa}$ (L50), SH3, and essential light chains (ELC) can be allocated in the map, along with part of the regulatory domain (RLC). Arrow heads indicate the interaction interfaces between actin and myosin at loop 4, helix-loop-helix motif, loop 3 of myosin (top to bottom). Arrow heads in the inset depict interaction interfaces at the cardiomyopathy loop and loop 2 (left to right). (C) Close-up of an actin subunit and structural model fitted into the EM map showing the four subdomains of an actin subunit (SD1-4). (D) Surface view of the structure of a complete myosin double-head including RLCs determined from averaging shifted sub-volumes. Their interface is indicated by a dotted line. (E) Comparison between the lower and upper heads within one double head, showing two different conformations in the lever arm that interacts with RLC and ELC. Lengths of the lever arms were measured between G772 and L844. (F) Alignment of the lower (purple) and upper (blue) heads heavy chain, showing two different kinks between the ELC-binding region and the RLC-binding region.

\section{References}

1. von Der Ecken, J., Heissler, S. M., Pathan-Chhatbar, S., Manstein, D. J. \&Raunser, S. Cryo-EM structure of a human cytoplasmic actomyosin complex at near-atomic resolution. Nature534, 724-728 (2016).

2. Mentes, A. et al. High-resolution cryo-EM structures of actin-bound myosin states reveal the mechanism of myosin force sensing. Proc. Natl. Acad. Sci. U. S. A.115, 1292-1297 (2018).

3. Ribeiro, E. D. A. et al. The structure and regulation of human muscle $\alpha$-Actinin. Cell159, 1447-1460 (2014).

4. Lombardi, V. et al. X-ray diffraction studies of the contractile mechanism in single muscle fibres. Philos. Trans. R. Soc. B Biol. Sci.359, 1883-1893 (2004).

5. AL-Khayat, H. A., Hudson, L., Reedy, M. K., Irving, T. C. \& Squire, J. M. Myosin head configuration in relaxed insect flight muscle: X-ray modeled resting cross-bridges in a pre-powerstroke state are poised for actin binding. Biophys. J.85, 1063-1079 (2003).

6. Squire, J. M., Roessle, M. \&Knupp, C. New X-ray diffraction observations on vertebrate muscle: Organisation of C-protein (MyBP-C) and troponin and evidence for unknown structures in the vertebrate A-band. J. Mol. Biol.343, 1345-1363 (2004).

7. Taylor, K. A., Reedy, M. C., Cordova, L. \& Reedy, M. K. Three-dimensional reconstruction of rigor insect flight muscle from tilted thin sections. Nature310, 285-291 (1984).

8. Schmitz, H. et al. Electron tomography of insect flight muscle in rigor and AMPPNP at $23^{\circ}$ C. J. Mol. Biol.264, 279-301 (1996).

9. Schmitz, H., Reedy, M. C., Reedy, M. K., Tregear, R. T. \& Taylor, K. a. Tomographic three-dimensional reconstruction of insect flight muscle partially relaxed by AMPPNP and ethylene glycol. J. Cell Biol.139, 695-707 (1997).

10. Liu, J. et al. Electron tomography of fast frozen, stretched rigor fibers reveals elastic distortions in the myosin crossbridges. J. Struct. Biol.147, 268-282 (2004). 